\title{
Planetary Nebulae: A Probe of the Galaxies Evolution up to the Hubble Time
}

\author{
A. F. Kholtygin ${ }^{1}$, and Yu. V. Milanova ${ }^{1}$ \\ ${ }^{1}$ Astronomical Institute of Saint-Petersburg, University, Russia \\ email: afk@astro.spbu.ru
}

Chemical evolution of the galactic and extragalactic planetary nebulae ( $\mathrm{PNe})$ system beginning from the early age of the Galaxy is investigated. We determine the radial and vertical abundance gradients for $\mathrm{C}, \mathrm{N}, \mathrm{O}, \mathrm{Ne}, \mathrm{Ar}, \mathrm{Cl}$ and $\mathrm{S}$ in a dependence on mass and age of the progenitor stars of the nebula. In the Table 1 we compare the galactic abundance gradients for $\mathrm{O}$ and $\mathrm{Ne}$ for our and neigbour galaxies.

We found the statistically significant dependence of abundance gradients for early ages of the Galaxy. The strong contradiction between the theoretical and obtained from observations gradients is found. A large number of PNe with extreemly low CNO abundances was detected. The dependence of the $\mathrm{C}, \mathrm{N}$ and $\mathrm{O}$ abundances in the $\mathrm{PNe}$ against the mass of their progenitor stars was investigated. The general accordance with syntetic model of the Galaxy chemical evolution Hoek and Groenewegen (1997) was found, excluding the case of $\mathrm{O}$, when the systematic lack of oxygen (about of two times) in a comparasion with Hoek and Groenewegen (1997) data was revealed.

We proposed the possibilty of two times enhanced infal rate for the Galaxy to explain the discordance between model and derived from the observed spectra of PNe element abundances.

Table 1. Radial composition gradient across the disks of spiral galaxies

\begin{tabular}{cccr}
\hline Galaxy & $\mathrm{d}[\mathrm{O} / \mathrm{H}] / \mathrm{dR}, \mathrm{dex} / \mathrm{kpc}$ & $\mathrm{d}[\mathrm{Ne} / \mathrm{H}] / \mathrm{dR}, \mathrm{dex} / \mathrm{kpc}$ & Reference \\
\hline M31 & -0.03 & - & Garnett et al. $(1997)$ \\
M33 & $-0.012 \pm 0.011$ & $-0.016 \pm 0.017$ & Crockett et al. $(2006)$ \\
M51 & -0.046 & - & Garnett et al. $(1997)$ \\
M81 & -0.08 & - & Garnett et al. $(1987)$ \\
M101 & $-0.028 \pm 0.01$ & - & Cedres et al. $(2004)$ \\
NGC2403 & $-0.102 \pm 0.009$ & - & Garnett et al. $(1997)$ \\
Milky Way & -0.012 & -0.019 & This work \\
\hline
\end{tabular}

\section{Acknowledgements}

This work has been supported by the Grant NSh-8542.2006.2 of the President of the Russian Federation for Leading Scientific schools.

\section{References}

Cedrés, B., Urbaneja, M.A., \& Cepa, J. 2004,A\&A 422, 511.

Crocket, K.C., Newton, R.J., Bottrell, S.H., \& Wignall, P.B. 2006, ApJ 637, 741.

Garnett, D.R. \& Shields, G.A. 1987, ApJ 317, 82.

Garnett, D.R., Shields, G.A., Skillman, E.D., Sagan, S.P., \& Dufour, R.J. 1997, ApJ 489, 63. van den Hoek, L.B., \& Groenewegen M.A.T. 1997, A\&SA Suppl. Ser. 123, 305. 